\title{
Iterated-greedy-based algorithms with beam search initialization for the permutation flowshop to minimise total tardiness
}

\author{
Victor Fernandez-Viagas ${ }^{\mathrm{a}, *}$, Jorge M.S. Valente ${ }^{\mathrm{b}}$, Jose M. Framinan ${ }^{\mathrm{a}}$ \\ a Industrial Management, School of Engineering, University of Seville, Camino de los Descubrimientos s/n, 41092 Seville, Spain \\ ${ }^{\mathrm{b}}$ LIAAD - INESC TEC, Faculdade de Economia, Universidade do Porto, Porto, Portugal
}

\section{A R T I C L E I N F O}

\section{Article history:}

Received 30 June 2017

Revised 15 September 2017

Accepted 23 October 2017

Available online 25 October 2017

\section{Keywords:}

Scheduling

Flowshop

Heuristics

PFSP

Tardiness

Beam search

Iterated greedy algorithm

Iterated local search

\begin{abstract}
A B S T R A C T
The permutation flow shop scheduling problem is one of the most studied operations research related problems. Literally, hundreds of exact and approximate algorithms have been proposed to optimise several objective functions. In this paper we address the total tardiness criterion, which is aimed towards the satisfaction of customers in a make-to-order scenario. Although several approximate algorithms have been proposed for this problem in the literature, recent contributions for related problems suggest that there is room for improving the current available algorithms. Thus, our contribution is twofold: First, we propose a fast beam-search-based constructive heuristic that estimates the quality of partial sequences without a complete evaluation of their objective function. Second, using this constructive heuristic as initial solution, eight variations of an iterated-greedy-based algorithm are proposed. A comprehensive computational evaluation is performed to establish the efficiency of our proposals against the existing heuristics and metaheuristics for the problem.
\end{abstract}

(c) 2017 Elsevier Ltd. All rights reserved.

\section{Introduction}

The flow shop is a common manufacturing layout in which a set of $n$ jobs has to be processed in a set of $m$ machines where each job follows the same route through the machines. For simplicity, the problem is denoted by permutation flow shop (PFSP in the following) when the same sequence of jobs is applied on each machine. The PFSP is one of the most studied optimization problems in Operations Research. Among the objectives studied in the literature (see e.g. Fernandez-Viagas, Dios, \& Framinan, 2016a; Fernandez-Viagas \& Framinan, 2014; Pan \& Ruiz, 2013), the minimisation of the total tardiness is essential for manufacturing systems (Raman, 1995), since due dates play an important role in these systems (Panwalkar, Smith, \& Seidmann, 1982) and delays may increase costs and/or the dissatisfaction of customers (resulting in either a poor reputation, or even the loss of customer) (Sen \& Gupta, 1984).

According to the $\alpha|\beta| \gamma$ notation (see e.g. Pinedo, 1995), the PFSP to minimise total tardiness can be denoted as Fm|prmu| $\Sigma T_{j}$. Since this problem is known to be NP-hard (Du \& Leung, 1990), during the last years several approximate algorithms -heuristics

\footnotetext{
* Corresponding author.

E-mail addresses: vfernandezviagas@us.es (V. Fernandez-Viagas), jvalente@fep.up.pt (J.M.S. Valente), vfernandezviagas@us.es (J.M. Framinan).
}

and metaheuristics- have been proposed in the literature (see e.g. Karabulut, 2016; Li, Chen, Xu, \& Gupta, 2015; Vallada, Ruiz, \& Minella, 2008). However, these proposals have not been compared against themselves, or the comparison has not been carried out under the same conditions, so the state-of-the-art regarding approximate algorithms for the problem remains unclear. Instead, these methods have been usually compared against either the genetic algorithm proposed by Vallada and Ruiz (2010), the Iterated Greedy (IG) algorithm by Ruiz and Stützle (2007), and/or the NEHedd by Kim (1993), which is the adaptation for the problem of the well-known NEH heuristic by Nawaz, Enscore Jr., and Ham (1983). The latter two are considered key methods in the flowshop scheduling literature since the noteworthy papers by Nawaz et al. (1983) and Ruiz and Stützle (2007), respectively. Regarding the NEHedd, it is probably the key constructive heuristic for the problem due to several reasons (FernandezViagas \& Framinan, 2015d): aside being an efficient heuristic for the problem, it is used to obtain an initial solution by the rest of efficient constructive or improvement heuristics, and by more than half of the efficient improvement heuristics or metaheuristics. Regarding IG, it remains the cornerstone of subsequent algorithms in the flowshop literature and can be considered as the state-of-the-art algorithm for several scheduling problems (see e.g. 
Fernandez-Viagas and Framinan, 2015b and Dubois-Lacoste, Pagnozzi, \& Stützle, 2017). ${ }^{1}$

Despite the preeminence of these two algorithms, recent advances in related scheduling problems have shown that they can be improved: On the one hand, some studies (see e.g. Dong, Huang, and Chen, 2009 and Pan \& Ruiz, 2014) have found better results by varying the destruction-construction phase in the IG for total flowtime minimisation, which is related to the problem under consideration (see Fernandez-Viagas \& Framinan, 2015d). On the other hand, recent constructive heuristics based on a noncomplete evaluation of the partial sequences have clearly outperformed the original NEH for other objective functions (see e.g. Fernandez-Viagas, Dios, et al., 2016a; Fernandez-Viagas \& Framinan, 2015c; 2017).

To tackle the aforementioned issues, the contribution of this work is twofold: We first implement a beam search algorithm for the problem which constructs several partial sequences in parallel. The algorithm estimates the value of the objective function for each partial sequence based on specific variables of the problem. We then develop several iterated-greedy-based algorithms to improve the pool of sequences generated by the beam search algorithm. To explore the effect of the construction phase in the algorithm, we implement eight different methods based on insertions, exchanges, randomness and optimizations of partial solutions. We finally compare the proposals with the best performing algorithms in the literature in an exhaustive computational evaluation.

The remainder of the paper is as follows: In Section 2 we formalise the problem and discuss its background. In Section 3 we propose the beam search and the iterated-greedy-based algorithms. These algorithms are compared with the state-of-the-art methods in Section 4. Finally, in Section 5 we discuss the main conclusions of the paper.

\section{Problem statement and background}

The problem under study can be set as follows: a set $\mathcal{N}$ of $n$ jobs have to be processed in a flowshop composed of a set $\mathcal{M}$ of $m$ machines. Each job $j \in\{1, \ldots, n\}$ has a due date $d_{j}$ and a processing time $p_{i j}$ on each machine $i \in\{1, \ldots, m\}$. Given a sequence of jobs $\Pi:=\left(\pi_{1}, \ldots, \pi_{r}, \ldots, \pi_{n}\right)$ where $r \in\{1, \ldots, n\}$ is an index of the position in a sequence, let $C_{i j}(\Pi)$ (abbreviated to $C_{i j}$ whenever it does not lead to confusion) be the completion time of job $j$ on machine $i$ according to sequence $\Pi$. Obviously, $C_{m j}(\Pi)$ is the completion time of job $j$ on the last machine, and $C_{m \pi_{n}}(\Pi)=C_{\max }$ is the maximum completion time or makespan of the sequence. The tardiness (earliness) of job $j$ is defined as $T_{j}=$ $\max \left\{C_{m j}-d_{j}, 0\right\}\left(E_{j}=\max \left\{d_{j}-C_{m j}, 0\right\}\right)$. Analogously, total tardiness, whose minimisation is the goal of our problem, is defined as $\sum T_{j}=\sum_{j=1}^{n} \max \left\{C_{m j}-d_{j}, 0\right\}$, while total earliness is defined as $\sum E_{j}=\sum_{j=1}^{n} \max \left\{d_{j}-C_{m j}, 0\right\}$. Note that the completion times can be computed recursively as follows:

$$
C_{i \pi_{j}}=\max \left\{C_{i-1, \pi_{j}}, C_{i, \pi_{j-1}}\right\}+p_{i \pi_{j}}
$$

where $C_{0 \pi_{j}}=C_{i \pi_{0}}=0$.

A number of approximate procedures have been proposed in the literature to provide good solutions for this problem in reasonable computation times. A review and evaluation of these algorithms prior to 2008 is given in Vallada et al. (2008). From this review, it turns out that the NEHedd proposed by Kim (1993), the ENS2 by Kim, Lim, and Park (1996), and the simulated annealing

\footnotetext{
${ }^{1}$ IG is currently a state-of-the-art algorithm for makespan minimisation $\left(F m|p r m u| C_{\max }\right)$. As stated by Fernandez-Viagas, Ruiz, and Framinan (2017), the speed up proposed by Taillard (1990) is probably one of main reason of the goodperformance of insertion phases -constructing jobs following a greedy method for that scheduling problem- as compared to randomized ones.
}

algorithms by Hasija and Rajendran (2004) and Parthasarathy and Rajendran (1997) (denoted as HR and SAH, respectively) are the most promising algorithms for the problem. Using the same computer conditions, Framinan and Leisten (2008) propose a hybrid algorithm (denoted as HA) which outperforms both the HR and the $\mathrm{SAH}^{\prime}$ (proposed by Parthasarathy \& Rajendran, 1998). ${ }^{2}$ This algorithm combines the iterated greedy and the variable neighbourhood search algorithms using a partial (adjacent-pairwiseexchange) local search in its construction phase, as well as an insertion local search improvement. In addition, Framinan and Leisten (2008) have also proposed a speed up mechanism to decrease the complexity of the evaluation. ${ }^{3}$ Recently, Karabulut (2016) have found around $50 \%$ time saving when applying it to the $\mathrm{NEH}$.

In parallel to the contribution by Framinan and Leisten (2008) and Vallada and Ruiz (2010) propose three genetic algorithms (GAPR, GAPR2, and GADV) that also outperform the HR and SAH in a fair comparison, and using a similar speed up mechanism for the problem. The best results were obtained by the GAPR version, although this algorithm was not compared to HA. Laterly, several contributions have outperformed the GAPR algorithm. First, Cura (2015) proposes an evolutionary algorithm (EA in the following), that outperforms both GAPR and SAH. The algorithm includes a mating procedure to diversify the solutions. Two local search methods with different neighbourhood sizes are employed, although the comparison is not performed using the same conditions, i.e. the algorithms used for comparison were not re-implemented. Secondly, several trajectory scheduling methods are proposed by Li et al. (2015) using six different composite heuristics (denoted as $\mathrm{CH}_{i}$ ) and three perturbation methods. Let us denote as $\mathrm{TSM}_{\mathrm{ij}}$ the trajectory scheduling methods composed of composite heuristic $\mathrm{CH}_{i}$ and perturbation method $j$. Among these methods, the best results are found by $\mathrm{TSM}_{63}$. On the one hand, under the same stopping criterion and the same computer conditions, $\mathrm{TSM}_{63}$ outperforms the three genetic algorithms by Vallada and Ruiz (2010), i.e. GAPR, GAPR2, and GADV. On the other hand, the proposed composite heuristics outperform the NEHedd but require additional CPU times. Regarding NEHedd, FernandezViagas and Framinan (2015d) analyse the structure of the problem, finding that there are a high number of ties in the selection procedure of NEHdd. Eight tie-breaking mechanisms are then proposed and compared with the original one, resulting that each tie-breaking mechanism (with the exception of the random one) statistically outperforms NEHedd. The most promising tie-breaking mechanisms are NEHedd $\left(\mathrm{TB}_{\mathrm{IT} 1}\right)$ and NEHedd $\left(\mathrm{TB}_{\mathrm{MS} \text {-Taillard, IT1 }}\right)$ (these heuristics are denoted in the following as $\mathrm{TB}_{\mathrm{IT} 1}$ and $\mathrm{TB}_{\mathrm{Ta}}$, respectively). In addition, they also statistically improve GAPR when used as an initial solution instead of the traditional NEHedd.

Recently, Karabulut (2016) propose KIG, an iterated greedy algorithm that incorporates a random local search method instead of the traditional insertion local search method. The search randomly performs insertion and exchanges using the speed up mechanism proposed by Framinan and Leisten (2008), until no more im-

\footnotetext{
2 This $\mathrm{SAH}^{\prime}$ algorithm was not included in the computational evaluation of Vallada et al. (2008) due to the resemblance of it with SAH.

${ }^{3}$ The speed up mechanism is a very common practice in the flowshop layout with permutation sequences. Taillard (1990) have proposed the first one for $\mathrm{Fm} \mid$ prmu $\mid C_{\max }$. Since then, several other mechanisms have been proposed for different constraints and/or objectives. Naderi and Ruiz (2010), Rios-Mercado and Bard (1998) and Fernandez-Viagas and Framinan (2015b) have successfully adapted them for the $D F|p r m u| C_{\max }, F m\left|s_{i j k}, p r m u\right| C_{\max }$ and $F m|p r m u| \epsilon\left(C_{\max } / T_{\max }\right)$ problems, respectively. With some modifications and lesser decrease in CPU times, they have been also adapted for the Fm $\mid$ prmu $\left|\Sigma C_{j}, F m\right| p r m u\left|\Sigma T_{j}, F m\right| p r m u \mid \sum E_{j}+\sum T_{j}$ and $F m \mid$ block $\mid \Sigma C_{j}$ problems by Li, Wang, and Wu (2009), Framinan and Leisten (2008), Fernandez-Viagas, Dios, et al. (2016a) and Fernandez-Viagas, Leisten, and Framinan (2016b), respectively.
} 
provement is achieved in $n$ iterations. In addition, it uses a simple annealing-like acceptance criterion with a constant temperature based on the makespan and the due dates of the jobs. Although the authors do not compare their proposal with algorithms specifically developed for the problem, they compare it with the original iterated greedy algorithm proposed by Ruiz and Stützle (2007) originally designed for the PFSP to minimise makespan-, which has been reimplemented for the problem under consideration. It is to note that such iterated greedy algorithm was found to be outperformed by GAPR for the problem under study.

To summarise, several algorithms have been proposed in the literature to solve the problem under consideration. However, the new picture of the efficient metaheuristics for the problem remains unclear due to the following issues:

1. The most promising metaheuristics found by Vallada et al. (2008) have been outperformed by GAPR and HA, but there is no comparison among these two latter algorithms and, to the best of our knowledge, contributions after 2008 do not include HA in their comparisons.

2. There is no computational comparison among the recent iterative algorithms proposed in the literature, i.e. EA, $\mathrm{TSM}_{63}$, and KIG.

3. Some metaheuristics are tested either under different computer conditions or versus non-state-of-the-art metaheuristics (see e.g. Cura, 2015; Karabulut, 2016).

In this paper, in Section 3 we first propose both a beam search algorithm with a fixed stopping criterion, and a set of eight different iterated-greedy-based algorithms varying their construction phase. In addition, we perform a comprehensive computational evaluation of the heuristics and metaheuristics in Section 4. By doing so, we establish the set of efficient algorithms for the problem.

\section{Proposed algorithms: beam search and iterated-greedy-based algorithms}

In this section, we propose several approximate procedures to solve the problem. The first proposal is a population-based constructive heuristic, which constructs several partial sequences in parallel, appending jobs, one by one, at the end of the sequences (see Section 3.1). Secondly, we propose several iterative improvement algorithms based on a single solution. Using the previous heuristic as initial solution, these algorithms iteratively search for the local optimum of a sequence obtained by a destructionconstruction-based phase (see Section 3.2). Note that the division between heuristics and metaheuristics is unclear in the literature and several classifications have been proposed (see e.g. Zanakis, Evans, \& Vazacopoulos, 1989; Zäpfel, Braune, \& Bögl, 2010). In this paper, we adopt the same definition as in Ruiz and Maroto (2005) and Fernandez-Viagas et al. (2017), where metaheuristics are defined as iterative improvement algorithms with stopping criteria depending on CPU time or number of iterations. In contrast, heuristics naturally stop when their steps are finished.

\subsection{Beam search algorithm}

In this subsection we present a beam search algorithm, denoted by $\operatorname{BS}(\gamma)$, with an advance priority evaluation function. Similarly to the $\mathrm{B} \& \mathrm{~B}$ algorithm, this approximate procedure constructs a search tree, where each node is formed by a partial sequence and the child nodes are obtained by adding one of the unscheduled jobs at the end of the parent node. However, only the most $\gamma$ promising nodes (denoted as beam width in the following) are kept for the next iteration. Beam search has been successfully applied to several scheduling problems in the literature (see e.g. Valente \&
Alves, 2005; 2008). Traditionally, two different functions have been applied to evaluate the nodes (Valente, 2010):

- Priority evaluation function. The node is evaluated by estimating the influence of the last job in the partial sequence. This evaluation of just one job in the node (omitting the influence of the other jobs) implies both that computing this function requires a low complexity order and that it is node-dependent, i.e. only children from the same parent node can be compared.

- Total cost evaluation function. The final objective function value to be achieved for this node is estimated taking into consideration all unscheduled jobs. Obviously, this function is nodeindependent as complete sequences are estimated. In addition, its complexity increases significantly.

Recently, Fernandez-Viagas, Leisten, et al. (2016b) and Fernandez-Viagas and Framinan (2017) have achieved excellent results using a node-independent priority evaluation function. The idea is to assign to each node a "genetic" code which keeps the historical behaviour of that node. By means of both this code and the influence of the last element, child nodes from different parents can be compared. This approach is applied in our proposal.

The proposed beam search algorithm is composed of several nodes in $n$ different levels. Let us denote by $S_{l}^{k}$ the partial sequence of the $l$ th node in iteration $k$, with $l \in[1, \gamma]$. Each node is then formed by a partial sequence $S_{l}^{k}, S_{l}^{k}:=\left(s_{1, l}^{k}, \ldots, s_{k, l}^{k}\right)$, of $k$ jobs, and by a set $\mathcal{U}_{l}^{k}$ (with $\mathcal{U}_{l}^{k}:=\left\{u_{1, l}^{k}, \ldots, u_{n-k, l}^{k}\right\}$ ) of $n-k$ unscheduled jobs. Whenever it does not lead to confusion, let us also denote that node by $S_{l}^{k}$. For each iteration $k, n-k$ child nodes are created from each partial sequence $S_{l}^{k}$ by adding one job from set $\mathcal{U}_{l}^{k}$ at the end of the sequence. The best $\gamma$ child nodes are selected to be the partial sequences of the nodes for the next iteration, i.e. $S_{l}^{k+1}$, $\forall l \in\{1, \ldots, \gamma\}$. More specifically, the steps of the proposed algorithm are as follows:

Step 1 Initialization

Step 2 While $k=2, \ldots, n-1$, repeat:

Step 2.1 Branching

Step 2.2 Node evaluation

Step 2.3 Node selection

Step 3 Final evaluation

To clarify both the branching and candidate selection phases, a simple example with five jobs and $\gamma=2$, i.e. $\mathrm{BS}(2)$, is shown in Fig. 1.

Next, each phase is explained in more detail:

- Initialization (Step 1). The first node is formed by job $j^{*}$ with the minimum sum of completion time on the last machine and weighted idle times, $w_{j}$. Let us denote such sum as index $\xi_{j}$, i.e. $j^{*}:=\arg \min _{j}\left\{\xi_{j}\right\}$ where:

$$
\begin{aligned}
\xi_{j}:=\sum_{i=1}^{m} p_{i j}+w_{j}= & \sum_{\substack{i=1 \\
\\
\forall j \in[1, n]}}^{m} p_{i j}+\frac{(n-2)}{4} \cdot \sum_{i=2}^{m}\left(\frac{m \cdot \sum_{i^{\prime}=1}^{i-1} p_{i^{\prime} j}}{i-1}\right),
\end{aligned}
$$

In case of ties, the job with minimum $w_{j}$ is chosen. See Liu and Reeves (2001), Fernandez-Viagas, Leisten, et al. (2016b), and Fernandez-Viagas and Framinan (2017) for similar initializations.

- Branching (Step 2.1). Each node $S_{l}^{k}$ is branched to form $n-k$ child nodes. Child node $v$ (with $v \in[1, n-k]$ ) is constructed from the node by adding $u_{v, l}^{k}$ at the end of the partial sequence $S_{l}^{k}$. Note that the number of child nodes in iteration $k$ is $\gamma(n-k)$. 


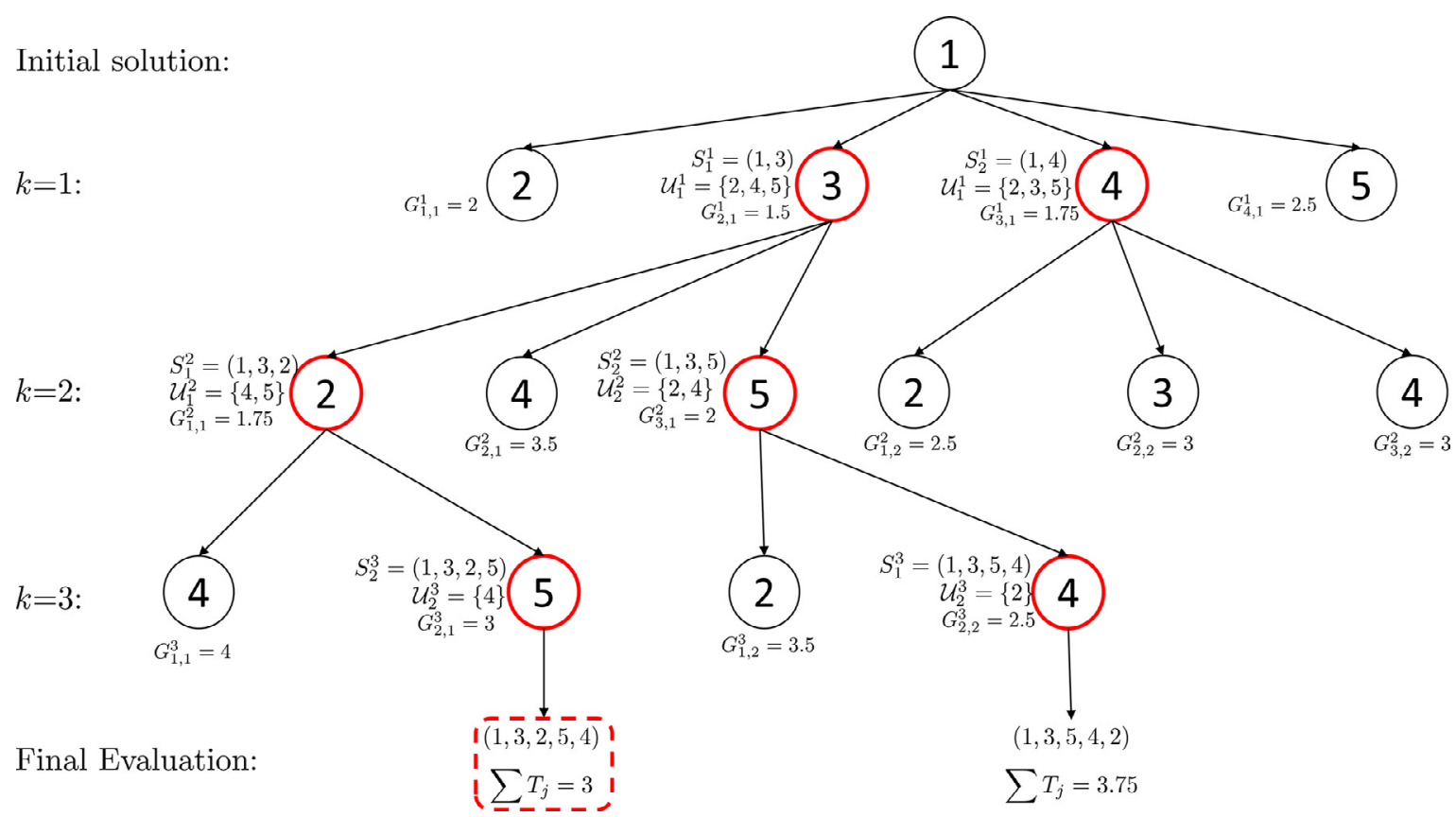

Fig. 1. Example of BS.

- Node evaluation (Step 2.2). As mentioned before, the child nodes are evaluated according to an index that weights two components:

- The "genetic" code. This first component measures the genetic offspring of the child node. By doing so, we are able to compare child nodes with different sequenced jobs, i.e. from different nodes. Obviously, each one of the child nodes of node $S_{l}^{k}$ has the same genetic code.

- The influence of the last element. It measures the influence of the last job inserted at the end of the partial sequence.

In order to define both influences, we explore the specific characteristics of the problem. Regarding the genetic code of node $S_{l}^{k}$, its goal is twofold. On the one hand, it should consider the contribution of the partial sequence to the objective function. As jobs in the following iterations are added always at the end of the partial sequence, the total tardiness of the jobs in the partial sequence stays unalterable until the end. On the other hand, it should address the indirect influences on the objective function of the future jobs to be added to the partial sequence. These influences make possible to compare child nodes of different offspring. To achieve these goals, the genetic code incorporates the following aspects which may be considered and balanced:

1. Cumulative total tardiness $(T T)$. It represents the total tardiness of partial sequences $S_{l^{\prime}}^{k+1}$. Note that $S_{I^{\prime}}^{k+1}$ represents the $l^{\prime}$ th best node of iteration $k+1$ formed by appending job $u_{v, l}^{k}$ at the end of node $S_{l}^{k}$. As $l$ and $l^{\prime}$ are not necessarily the same nodes, let us denote by job[l'] such job $u_{v, l}^{k}$ and by branch $\left[l^{\prime}\right]$ such $l$. Then the cumulative total tardiness of $S_{l^{\prime}}^{k+1}$ is computed as follows:

$$
\begin{aligned}
T T_{l^{\prime}}^{k+1} & =T T_{\text {branch }\left[l^{\prime}\right]}^{k}+T_{j o b\left[l^{\prime}\right], \operatorname{branch}\left[l^{\prime}\right]}^{k}, \quad \forall k=\{2, \ldots, n-1\}, \\
l^{\prime} & =\{1, \ldots, \gamma\}
\end{aligned}
$$

where $T_{j l}^{k}$ is the tardiness of job $j$ of node $S_{l}^{k}$ in iteration $k$.

2. Cumulative total earliness (TE). Analogously, it represents the total earliness of partial sequences $S_{l^{\prime}}^{k+1}$. Denoting by $E_{j l}^{k}$ the earliness of job $j$ of node $S_{l}^{k}$ in iteration $k$, the cumulative total earliness of node $S_{l^{\prime}}^{k+1}$ in iteration $k+1$ is defined as follows:

$$
\begin{aligned}
T E_{l^{\prime}}^{k+1} & =T E_{\text {branch }\left[l^{\prime}\right]}^{k}+E_{j o b\left[l^{\prime}\right], \operatorname{branch}\left[l^{\prime}\right]}^{k}, \quad \forall k=\{2, \ldots, n-1\}, \\
l^{\prime} & =\{1, \ldots, \gamma\}
\end{aligned}
$$

3. Cumulative weighted idle time (TI). It represents the cumulative weighted idle time of each job of partial sequence $S_{l^{\prime}}^{k+1}$, which is defined by:

$$
\begin{aligned}
T I_{l^{\prime}}^{k+1} & =T I_{\text {branch }\left[l^{\prime}\right]}^{k}+I_{j o b\left[l^{\prime}\right], \operatorname{branch}\left[l^{\prime}\right]}^{k}, \quad \forall k=\{2, \ldots, n-1\}, \\
l^{\prime} & =\{1, \ldots, \gamma\}
\end{aligned}
$$

where $I_{j l}^{k}$ is the weighted idle time between the last job of sequence $S_{l}^{k}$ and job $j$, which is inserted in the last position of the sequence, see Eq. (6).

$$
I_{j l}^{k}=\sum_{i=2}^{m} \frac{m \cdot \max \left\{C_{i-1, j}-C_{i,[k]}, 0\right\}}{i-1+(k-1) \cdot(m-i+1) /(n-2)}
$$

Note that, after the node selection phase, the cumulative total tardiness, total earliness and weighted idle time are updated by incorporating the corresponding value of the last job. Thereby, it avoids to completely re-calculate of them in each iteration. Obviously, the influence of the cumulative total tardiness increases when the sequence contains more jobs, while the weight of the indirect influence (i.e. the cumulative total earliness and idle time) decreases with each iteration. More specifically, in the first iterations of the algorithm, it seems better to choose sequences with high values of total idle times and total earliness times to have more promising partial sequences for the next iterations. In contrast, in the last iterations, the influence of the total tardiness becomes more relevant as objective function of the problem. Among TE and TI, the total earliness seems to be a better estimation of the tardiness of the unscheduled jobs in these last iterations. In addition, by keeping $T E$ in the last iterations, we break the greedy behaviour of the total tardiness. To deal with these issues and after some preliminary tests, we propose weights for the three components following the simple linear functions shown in Fig. 2. 


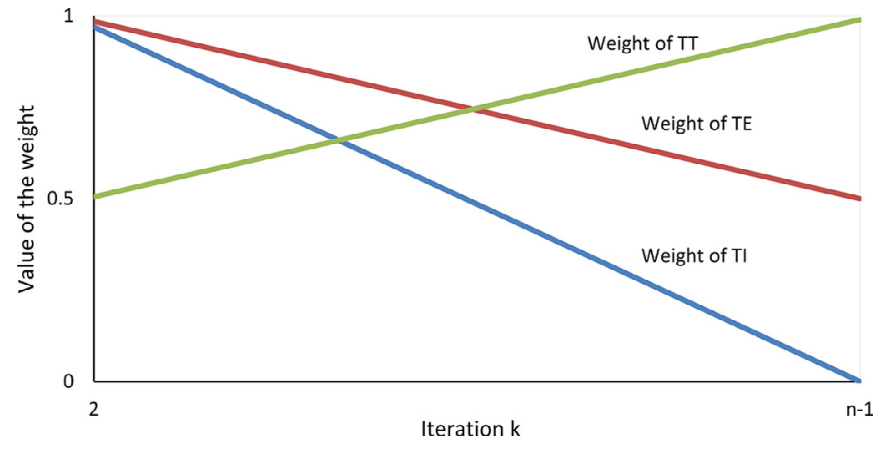

Fig. 2. Values of the weights of total tardiness, total earliness, and total weighted idle time, depending of the iteration $k$.

Hence, the genetic code, denoted as $F_{l^{\prime}}^{k}$, of node $l^{\prime}$ in iteration $k$ has the following expression:

$$
\begin{aligned}
F_{l^{\prime}}^{k} & =T I_{l^{\prime}}^{k} \cdot \frac{n-k-1}{n}+a \cdot T E_{l^{\prime}}^{k} \cdot \frac{2 n-k-1}{2 n}+b \cdot T T_{l^{\prime}}^{k} \cdot \frac{k-1+n}{2 n}, \\
\forall k & =\{2, \ldots, n-1\}, \quad l^{\prime}=\{1, \ldots, \gamma\}
\end{aligned}
$$

where $a$ and $b$ are parameters of the algorithm to balance the contribution of TI, TE and TT. Implicitly, low values for $a$ and $b$ indicate that the contribution of $T I$ is higher than $T E$ and TT. So, in order to reduce the number of parameters of the algorithm, only $a$ and $b$ (for $T E$ and $T T$, respectively) are considered, and the influence of $T I$ is measured varying this parameter as $T I$ was normalized.

Regarding the index, denoted as $L_{v l}^{k}$, employed to estimate the contribution of the last job, $u_{v l}^{k}$, when evaluating child node $v$ of node $S_{l}^{k}$, we follow a similar procedure to the genetic code, where the weighted idle time and the earliness time are chosen as criteria. Note that the influence of the tardiness of the last job is included in the earliness since a tardy job indicates an earliness equals to 0 . In addition, once several jobs are tardy, they stay tardy in the following iterations and the influence of other elements should be taken into account to choose the job. Regarding these other elements, several studies (see e.g. Fernandez-Viagas \& Framinan, 2015c; Liu \& Reeves, 2001) found excellent results by incorporating an estimation of the contribution of the unscheduled jobs. To deal with that, we add the total tardiness of all jobs $u_{v l}^{k}, \forall v \in 1, \ldots, n-k$, denoted by $W_{l}^{k}$. Note that job $u_{v l}^{k}$ is also included in $W_{l}^{k}$ index of child node $v$. By doing so, we reduce the computational effort of this index. Hence, index $L_{v l}^{k}$ can be defined by Expression (8), where $c$ and $e$ are parameters of the algorithm again to balance the contributions of $E_{u_{v l}^{k}, l}^{k}$ and $W_{l}^{k}$. Similarly to $F_{l^{\prime}}^{k}$, after a preliminary test, we use a decreasing function for the weight of the idle time, and an increasing one for $W_{l}^{k}$ (the tardiness of the unscheduled jobs is closer to the real one in the last iterations than in the first ones).

$$
\begin{aligned}
L_{v l}^{k} & =(n-k-1) \cdot I_{u_{v l}^{k} l}^{k}+c \cdot E_{u_{v l}^{k}, l}^{k}+\frac{e}{n-k+1} \cdot W_{l}^{k}, \\
\forall k & =\{2, \ldots, n-1\}, \quad l=\{1, \ldots, \gamma\}, \quad v=\{1, \ldots, n-k\}
\end{aligned}
$$

Then, each child node $v$ obtained by node $S_{l}^{k}$ is computed using index $G_{v l}^{k}$ which adds both contributions:

$$
\begin{aligned}
G_{v l}^{k} & =F_{l}^{k}+L_{v l}^{k}, \quad \forall k=\{2, \ldots, n-1\}, \quad l=\{1, \ldots, \gamma\}, \\
v & =\{1, \ldots, n-k\}
\end{aligned}
$$

- Node selection (Step 2.3). Among the $\gamma(n-k)$ child nodes in iteration $k$, the best $\gamma$ ones are kept as the set of nodes of iteration $k+1$. More specifically, in iteration $k$, the $\gamma$ nodes with the lowest values of the $G_{v l}^{k}$ indicator $(\forall v \in\{1, \ldots, n-k\}, l \in$ $\{1, \ldots, \gamma\})$ are selected for the next iteration.

- Final evaluation (Step 3). The total tardiness of the nodes selected in the last iteration, i.e. nodes $S_{l}^{n-1}(\forall l \in 1, \ldots, \gamma)$, is evaluated. The sequence yielding the minimal total tardiness is the final sequence of the beam search algorithm.

\subsection{Iterated-greedy-based algorithms, IA}

The iterated greedy algorithm is a single-solution-based metaheuristic, originally proposed for flow-shop-type scheduling problems by Ruiz and Stützle (2007). Starting with an initial solution, this metaheuristic iteratively perturbs a sequence and searches for its local optimum. Then, the iterated greedy algorithm destructs several jobs of a sequence in each iteration, and constructs them following a greedy approach. More specifically, in a destruction phase, $d$ jobs are randomly removed from the iteration sequence, denoted as $\Pi^{i}$. Let us denote by $\Pi^{r}, \Pi^{r}:=\left(\pi_{1}^{r}, \ldots, \pi_{d}^{r}\right)$, the sequence formed by the removed jobs and by $\Pi^{d}$ the partial sequence of length $n-d$, formed by $\Pi^{i}$ without the removed jobs. After that, in a construction phase, each job in $\Pi^{r}$ is inserted in the position of $\Pi^{d}$ yielding the lowest value of the objective function. $\Pi^{c}$ is the so obtained sequence. Finally, this metaheuristic looks for the local optimum of the constructed sequence and performs a basic simulated annealing phase. In this subsection we propose eight simple Iterated Algorithms based on the iterated greedy metaheuristic, ${ }^{4}$ denoted as IA, consisting on the following same four phases which are repeated until a stopping criterion is reached: destruction phase; construction phase; local search phase; and a simple simulated annealing phase. As described earlier, the destruction-construction phase plays an important role in the efficiency of the metaheuristic. In order to take it into account, in this paper we propose and compare the following eight different procedures:

1. Random insertion (let us denote by $I_{\mathrm{RI}}$ the proposed algorithm using this construction phase) This procedure replaces the greedy insertion of the traditional iterated greedy by a random one. More specifically, each removed job $\pi_{i}^{r}, \forall i \in 1, \ldots, d$, is randomly inserted in $\Pi^{d}$.

2. Greedy insertion $\left(\mathrm{IA}_{\mathrm{GI}}\right)$. This is the traditional construction phase of the iterated greedy algorithm, i.e. each removed job $\pi_{i}^{r}, \forall i \in 1, \ldots, d$, is inserted in the position of $\Pi^{d}$ yielding the lowest total tardiness.

3. Random general swap ( $\left.I A_{R G S}\right)$. This procedure replaces the destruction and construction phase of the algorithm by performing $d$ random exchanges between jobs, i.e., $d$ jobs are randomly chosen from $\Pi^{i}$ and exchanged with other jobs of this sequence.

4. Greedy general swap $\left(\mathrm{IA}_{\mathrm{GGS}}\right)$. In this procedure, a job is randomly chosen from $\Pi^{i}$ and exchanged with each job of the sequence. Sequence $\Pi^{i}$ is replaced by the exchange yielding the lowest total tardiness. The procedure is repeated $d$ times for $d$ different jobs.

5. Random adjacent swap ( $\left(\mathrm{A}_{\mathrm{RAS}}\right)$. This procedure randomly chooses a job and exchanges it with the next job of the sequence. The procedure is repeated $d$ times.

\footnotetext{
4 Note that the iterated greedy algorithm is closely related to the iterated local search and in fact, it could be considered as a special case of the iterated local search. This latter algorithm begins with an initial solution and iteratively modifies the current solution and looks for its local optimum. Therefore, assuming that a special type of modification of a solution is to perform the destruction and construction phase of the iterated greedy, both algorithms would be considered the same metaheuristic. However, in order to maintain the coherence with previous proposals in the literature, we also use the term "iterated-greedy-based algorithm" in the notation of our proposals.
} 
6. Greedy insertion + Partial adjacent-swap-based local search method ( $\left.\mathrm{IA}_{\mathrm{GI} \text { ALS }}\right)$. This procedure is based on Framinan and Leisten (2008). Thereby, each job removed $\pi_{i}^{r}, \forall i \in\{1, \ldots, d\}$ is inserted in the position of $\Pi^{d}$ yielding the lowest total tardiness (denoted as position $b$ ), as in the greedy insertion procedure. After that, an adjacent pairwise exchange is performed for the jobs between the last position of the partial sequence and position $b+1$.

7. Insertion-based Local search + Greedy insertion $\left(\mathrm{IA}_{\text {ILS_GI }}\right)$. This procedure adapts the procedure of destruction and construction proposed by Dubois-Lacoste et al. (2017). The method performs an insertion-based local search on the partial sequence $\Pi_{d}$, i.e. each job of the sequence is removed and inserted in the best position. The procedure is repeated until there is no improvement in a complete iteration. The best sequence found by the algorithm replaces $\Pi_{d}$. After that, the traditional construction phase (i.e. greedy insertion) is applied.

8. Greedy insertion + Local search insertion $\left(\mathrm{IA}_{\mathrm{GI} \_I L S}\right)$. This procedure is an adaptation of the method proposed by Pan and Ruiz (2014) and Pan, Gao, Li, and Gao (2017). Similarly as $\mathrm{IA}_{\mathrm{GI} \_\mathrm{ALS}}$, each removed job $\pi_{i}^{r}, \forall i \in\{1, \ldots, d\}$ is inserted in the position $b$ of $\Pi^{d}$ yielding the lowest total tardiness. After that, jobs in positions $b-1$ and $b+1$ are removed and reinserted in the position yielding the lowest total tardiness.

After the destruction-construction procedure, a local optimum of sequence $\Pi^{c}$ is obtained in the local search phase. This phase iteratively removes each job in sequence $\Pi^{c}$ and inserts it in the position with the lowest vaue of the objective function. The phase is stopped after a complete iteration without any improvement. Finally, the simulated annealing-like acceptance criterion proposed by Karabulut (2016) is applied due to its excellent performance. This simple criterion is a variation of the proposal of Ruiz and Stützle (2007) for $F m|p r m u| C_{\max }$, which has been successfully applied to other several objectives and/or scheduling problems (see e.g. Fernandez-Viagas \& Framinan, 2015a; 2015b; Ribas, Companys, \& Tort-Martorell, 2017). The criterion uses a constant Temperature which depends on parameter $T$ of the algorithm:

$$
\text { Temperature }=T \cdot \frac{\sum_{j=1}^{n}\left(L B_{C_{\max }}-d_{j}\right)}{n \cdot 10}
$$

where $L B_{C_{\max }}$ is the lower bound of the makespan following the procedure established by Taillard (1993). The pseudo-code of the proposed metaheuristic is shown in Fig. 3. Note that BS is used as the initial solution of the proposed iterated-greedy-based algorithms.

\section{Computational experience}

In this section we compare the state-of-the-art algorithms against our proposals. Prior to performing this computational evaluation, we establish the conditions adopted to achieve a fair comparison. Firstly, in Section 4.1 we present the sets of instances generated. Secondly, the measures to evaluate both the quality of the solutions and the computational requirements of each algorithm are shown in Section 4.2. Regarding our proposals, two full experimental parameter tunings are described in Section 4.3. Next, in Section 4.4, the state-of-the-art algorithms, which are fully reimplemented, are shown. We compare them against our proposals by carrying out two different computational evaluations for heuristics and metaheuristics, see Sections 4.5 and 4.6, respectively.

\subsection{Sets of instances}

In this paper, two benchmark testbeds, denoted as $\beta_{1}$ and $\beta_{2}$, are generated for the experiments of our study. $\beta_{1}$ is used for the calibration of the parameters of the proposed algorithms. The computational evaluations of both heuristics and metaheuristics are carried out on benchmark $\beta_{2}$. By doing so, we avoid an over calibration of the parameters of our algorithms in the benchmark of comparison.

- Benchmark $\beta_{1}$ : This benchmark is generated by the procedure described in Vallada and Ruiz (2010). It contains 108 different sizes of the problem varying the parameters $n, m, T$ and $R$. Ten instances are generated for each combination of parameters $n \in\{50,150,250,350\}, m \in\{10,30,50\}, T \in\{0.2,0.4,0.6\}$, and $R \in\{0.2, .0 .6,1.0\}$, i.e. a total of 1080 instances are generated in this benchmark. $T$ and $R$ are parameters to generate different types of due dates for each size of the problem (see Potts \& Van Wassenhove, 1982). They generate the processing times and the due dates with a uniform distribution [1, 99] and $[P \cdot(1-T-R / 2, P \cdot(1-T+R / 2]$, respectively, where $P$ is the lower bound for the makespan proposed in Taillard (1993).

- Benchmark $\beta_{2}$ : This benchmark is composed of the 540 instances of Vallada et al. (2008). It contains 108 combinations of parameters $n \in\{50,150,250,350\}, m \in\{10,30,50\}, T \in\{0.2,0.4$, $0.6\}$, and $R \in\{0.2, .0 .6,1.0\}$, with five instances for each combination. Processing times and due dates are generated following the same distributions than in benchmark $\beta_{1}$.

\subsection{Performance indicators}

In our study, two computational evaluations are carried out to compare the most promising heuristics and metaheuristics. As a result, 23 algorithms are tested. To conduct a fair comparison among them, the algorithms are compared under the same conditions. More specifically, the following aspects are considered:

- We use the same computer (an Intel Core i7-3770 with $3.4 \mathrm{GHz}$, 16GB RAM, and with Microsoft Windows 8.1 64 bit operating system).

- We re-code each algorithm using the same programming language (C\# under Visual Studio 2013).

- We use the same computational skills, libraries and common functions.

- We use the same stopping criteria for each metaheuristic.

In addition, each algorithm typically requires a different CPU time and obtains a different solution. In order to compare both the quality of the solutions and the computational efforts of the implemented algorithms, the indicators for comparison have to be established. On the one hand, heuristics are compared using the Average Relative Deviation Index (denoted as $A R D I 1_{h}$ for heuristic $h$ ) and the Average Relative Percentage computation Time (denoted as $A R P T_{h}$ for heuristic $h$ following the recommendation established by Fernandez-Viagas and Framinan (2015c) and FernandezViagas et al. (2017) (see Eqs. (11) and (12), respectively). On the other hand, metaheuristics are only compared using the $A R D I 1_{h}$ as the same CPU times are used.

$$
\begin{gathered}
A R D I 1_{h}=\sum_{i=1}^{I} \frac{R D I 1_{i h}}{I}, \quad \forall h=1, \ldots, H \\
A R P T_{h}=1+\sum_{i=1}^{I} \frac{R P T_{i h}}{I}, \quad \forall h=1, \ldots, H
\end{gathered}
$$

Let $I$ be the number of instances, and $H$ be the number of considered heuristics. The Relative Deviation Index of heuristic $h$ in instance $i, R D I 1_{i h}$, and the Relative Percentage computation Time, $R P T 1_{i h}$, are defined by the following expressions, respectively:

$$
R D I 1_{i h}=\frac{\text { OF }_{\text {ih }}-\text { Best }_{i}}{\text { Worst }_{i}-\text { Best }_{i}}, \quad \forall i=1, \ldots, I, h=1, \ldots, H
$$




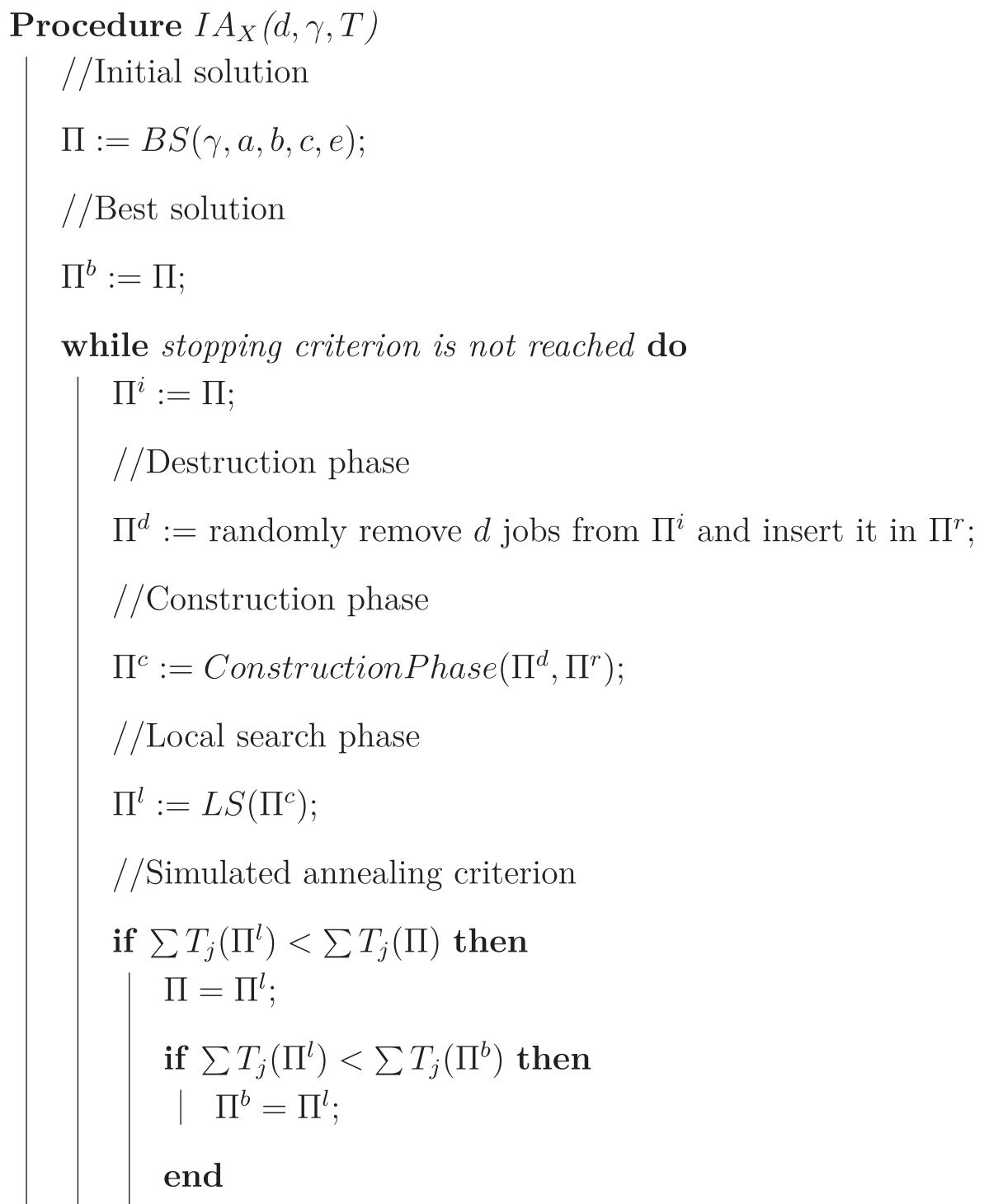

else if random $\leq \exp \left\{-\left(C_{\max }\left(\pi^{l}\right)-C_{\max }(\pi)\right) /\right.$ Temperature $\}$ then

$$
\Pi=\Pi^{l}
$$

end

end

end

Fig. 3. Proposed iterated-greedy-based algorithms.

$$
R P T_{i h}=\frac{T_{\text {ih }}-A C T_{i}}{A C T_{i}}, \quad \forall i=1, \ldots, I, h=1, \ldots, H
$$

where Best $_{i}$ and Worst $_{i}$ are the best and worst known solution for one run in instance $i^{5}$, respectively. Let $T_{i h}$ and $O F_{i h}$ be the CPU time and the objective function value obtained by heuristic $h$ in iteration $i$, respectively. Finally, $A C T_{i}$ is the average CPU time required by all compared algorithms in iteration $i$, which is defined

5 These values are presented as on-line materials, which are taken from http: //soa.iti.es/problem-instances. by:

$$
A C T_{i}=\frac{\sum_{h=1}^{H} T_{i h}}{H}, \quad \forall i=1, \ldots, I
$$

Regarding the experimental parameter tuning on benchmark $\beta_{1}$, we apply a different indicator of the quality of the solution. More specifically, we used $A R D I 2$, which is a small modification of ARDI1:

$$
A R D I 2_{h}=\sum_{i=1}^{I} \frac{R D I 2_{i h}}{I}, \quad \forall h=1, \ldots, H
$$


Table 1

Average results of RDI2 for each tested parameter.

\begin{tabular}{|c|c|c|c|c|c|c|c|c|c|c|c|}
\hline \multicolumn{2}{|c|}{ Parameter $a$} & \multicolumn{2}{|c|}{ Parameter $b$} & \multicolumn{2}{|c|}{ Parameter $c$} & \multicolumn{2}{|c|}{ Parameter $e$} & \multicolumn{2}{|c|}{ Parameter $d$} & \multicolumn{2}{|c|}{ Parameter $\gamma$} \\
\hline Level & ARDI2 & Level & ARDI2 & Level & ARDI2 & Level & ARDI2 & Level & ARDI2 & Level & ARDI2 \\
\hline 0.00 & 27.68 & 0.00 & 27.59 & 0.25 & 43.23 & 2 & 30.98 & 4 & 36.75 & 2 & 54.36 \\
\hline 0.25 & 28.37 & 0.15 & 26.22 & 0.50 & 30.65 & 3 & 29.17 & 5 & 39.84 & $n / 10$ & 32.22 \\
\hline 0.50 & 28.27 & 0.30 & 34.29 & 0.75 & 26.08 & 4 & 28.61 & 6 & 42.82 & $n / m$ & 42.85 \\
\hline 0.75 & 29.33 & & & 1.00 & 24.93 & 5 & 28.72 & & & 10 & 38.50 \\
\hline 1.00 & 30.70 & & & 1.25 & 24.67 & & & & & 15 & 35.55 \\
\hline 1.25 & 32.14 & & & 1.50 & 27.05 & & & & & $n$ & 35.33 \\
\hline
\end{tabular}

Table 2

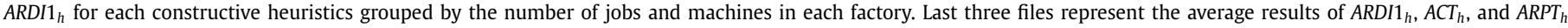
for constructive heuristics.

\begin{tabular}{|c|c|c|c|c|c|c|c|c|c|c|c|c|c|c|c|c|}
\hline$n \times m$ & NEHedd & $\mathrm{TB}_{\mathrm{Ta}}$ & $\mathrm{TB}_{\mathrm{IT} 1}$ & $\mathrm{CH} 1$ & $\mathrm{CH} 2$ & $\mathrm{CH} 3$ & $\mathrm{CH} 4$ & CH5 & $\mathrm{CH} 6$ & $\mathrm{BS}(2)$ & $\mathrm{BS}(5)$ & $\mathrm{BS}(10)$ & $\mathrm{BS}(15)$ & $\mathrm{BS}(n / 10)$ & $\mathrm{BS}(n / m)$ & $\mathrm{BS}(n)$ \\
\hline $50 \times 10$ & 17.46 & 14.53 & 13.72 & 6.26 & 7.72 & 5.30 & 5.35 & 5.88 & 5.46 & 15.29 & 11.20 & 10.70 & 10.22 & 11.20 & 11.20 & 16.52 \\
\hline $50 \times 30$ & 19.79 & 18.68 & 18.61 & 11.12 & 14.43 & 9.84 & 10.17 & 10.51 & 10.40 & 24.26 & 19.11 & 15.90 & 15.95 & 19.11 & 30.13 & 25.09 \\
\hline $50 \times 50$ & 18.17 & 17.97 & 17.57 & 10.94 & 14.34 & 10.66 & 10.99 & 10.61 & 10.75 & 22.96 & 18.47 & 16.14 & 15.78 & 18.47 & 29.15 & 26.86 \\
\hline $150 \times 10$ & 13.80 & 10.69 & 9.91 & 3.67 & 4.14 & 2.86 & 2.87 & 3.04 & 2.82 & 8.89 & 6.17 & 5.22 & 5.58 & 5.58 & 5.58 & 8.96 \\
\hline $150 \times 30$ & 20.70 & 17.02 & 15.81 & 8.21 & 10.35 & 7.51 & 7.20 & 8.04 & 7.15 & 18.93 & 11.23 & 9.22 & 8.50 & 8.50 & 11.23 & 12.55 \\
\hline $150 \times 50$ & 22.04 & 19.64 & 18.57 & 9.62 & 12.57 & 9.02 & 8.83 & 9.15 & 8.75 & 23.93 & 15.90 & 12.56 & 10.70 & 10.70 & 19.74 & 16.98 \\
\hline $250 \times 10$ & 10.06 & 7.26 & 6.70 & 2.23 & 1.97 & 1.28 & 1.08 & 1.81 & 1.37 & 6.51 & 4.81 & 4.11 & 4.14 & 4.14 & 4.14 & 7.33 \\
\hline $250 \times 30$ & 17.81 & 13.29 & 11.62 & 4.72 & 6.10 & 4.38 & 4.05 & 4.37 & 4.17 & 13.33 & 8.02 & 5.43 & 4.48 & 3.75 & 6.76 & 6.80 \\
\hline $250 \times 50$ & 20.21 & 15.90 & 13.96 & 6.20 & 8.76 & 5.84 & 5.67 & 5.95 & 6.02 & 19.28 & 11.76 & 8.68 & 6.97 & 5.36 & 11.76 & 8.86 \\
\hline $350 \times 10$ & 9.01 & 6.65 & 6.14 & 1.98 & 1.12 & 0.80 & 0.67 & 1.06 & 0.69 & 4.81 & 2.76 & 2.45 & 2.31 & 2.49 & 2.49 & 4.91 \\
\hline $350 \times 30$ & 15.74 & 11.40 & 9.84 & 3.34 & 3.95 & 2.65 & 2.38 & 3.09 & 2.49 & 10.26 & 5.07 & 3.27 & 2.52 & 1.58 & 3.04 & 3.82 \\
\hline $350 \times 50$ & 17.38 & 13.11 & 11.10 & 3.99 & 5.78 & 3.50 & 3.53 & 3.83 & 3.61 & 15.68 & 9.52 & 6.27 & 5.43 & 3.18 & 8.31 & 4.84 \\
\hline$A R D I 1_{h}$ & 16.85 & 13.84 & 12.80 & 6.02 & 7.60 & 5.30 & 5.23 & 5.61 & 5.31 & 15.34 & 10.34 & 8.33 & 7.71 & 7.84 & 11.96 & 11.96 \\
\hline$A C T_{h}$ & 1.56 & 1.53 & 1.56 & 119.94 & 10.01 & 66.74 & 63.78 & 139.59 & 88.35 & 0.05 & 0.12 & 0.26 & 0.40 & 0.84 & 0.27 & 17.16 \\
\hline$A R P T_{h}$ & 0.13 & 0.13 & 0.13 & 2.93 & 0.41 & 2.13 & 2.07 & 3.63 & 2.51 & 0.01 & 0.03 & 0.06 & 0.10 & 0.08 & 0.04 & 1.60 \\
\hline
\end{tabular}

$$
R D I 2_{i h}=\frac{O F_{i h}-\text { Best }_{i}^{\prime}}{\text { Wrst }_{i}^{\prime}-\text { Best }_{i}^{\prime}}, \quad \forall i=1, \ldots, I, h=1, \ldots, H
$$

where Best $_{i}^{\prime}$ and Worst ${ }_{i}^{\prime}$ are the best and worst total tardiness among the algorithms tested in the calibration, respectively.

\subsection{Experimental parameter tuning}

In this subsection, two full factorial design of experiments are presented to determine the best combinations of parameters for the proposed algorithms. Both experiments are evaluated on benchmark $\beta_{1}$. Regarding BS, firstly four parameters ( $a, b, c$ and $e$ ) have been proposed to balance the contributions in the evaluation of partial sequences. In addition, parameter $\gamma$ (beam width) directly influences its complexity, $O\left(\max \left\{\gamma \cdot n^{2} \cdot m, \gamma^{2} \cdot n^{2}\right\}\right)$, and consequently the CPU time of the proposed beam search. For each value of $\gamma$, there is a trade-off between the quality of solutions and the computational effort. Thus, this parameter is removed of this experimental parameter tuning (see e.g. Fernandez-Viagas and Framinan, 2015c; Fernandez-Viagas, Leisten, et al., 2016b; Liu and Reeves, 2001 for similar approaches) to avoid a calibration of each parameter $\gamma$, and its value is set to 15 . So the following levels of the parameters are tested:

$$
\begin{aligned}
& \text { - } a \in\{0,0.25,0.5,0.75,1,1.25\} \\
& \text { - } b \in\{0,0.15,0.3\} \\
& \text { - } c \in\{0.25,0.5,0.75,1,1.25,1.5\} \\
& \text { - } e \in\{2,3,4,5\}
\end{aligned}
$$

Regarding the proposed iterated-greedy-based algorithms, they use three parameters: $d, \gamma$, and $T$. Firstly, we use in this test $d \in\{4$, $5,6\}$ for the number of destructed jobs. Regarding the parameter $\gamma$, the CPU time of $\mathrm{IA}_{\mathrm{i}}$ depends on its stopping criterion instead of $\gamma$, since BS is applied as its initial solution. So, different values of $\gamma$ only perturbs its objective function value, i.e. we may now measure the influence of $\gamma$ in the quality of the solutions of the metaheuristics without altering its CPU time. In this calibration test, we use the following levels, $\gamma \in\{2, n / 10, n / m, 10,15, n\}$. For parameter $T$, we use the best value found by Karabulut (2016), i.e. $T=1.0$, since its influence has not been found to be statistically significant in several previous studies (see e.g. Fernandez-Viagas \& Framinan, 2015a; Pan \& Ruiz, 2014). The calibration test is carried out for $I A_{R I}$ and using $n \cdot(m / 2) \cdot 60 \mathrm{~ms}$.

In this paper, we carry out two non-parametric Kruskal-Wallis analyses to determine the statistical differences between the levels of the parameters. Note that the normality and homoscedasticity assumptions were not satisfied. In addition, the indicator ARDI2 has been used to evaluate the quality of the solutions. The results show that there are statistically significant differences between the level of each parameter $(a, b, c, e, d$, and $\gamma)$, since each $p$-value obtained in the tests is .000 . The best combination of parameters has been found for $a=0, b=0.15, c=1.25, e=4, d=4$, and $\gamma=n / 10$. These values of the parameters are used in the next sections. The average results for each level of the parameters, in terms of ARDI2, are shown in Table 1.

\subsection{Implemented algorithms}

The proposed algorithms, $\mathrm{BS}$ and $\mathrm{IA}_{\mathrm{i}}$, are compared against the state-of-the-art algorithms in two different computational evaluations. Following the discussion in Section 2, the following heuristics and metaheuristics are implemented in this study:

- Heuristics

- NEHedd proposed by Kim (1993).

- $\mathrm{TB}_{\mathrm{IT} 1}$ and $\mathrm{TB}_{\mathrm{Ta}}$ proposed by Fernandez-Viagas and Framinan (2015d).

- $\mathrm{CH}_{i} \forall i=1, \ldots, 6$ proposed by Li et al. (2015).

- The BS $(\gamma)$ algorithms proposed in Section 3.1, with $\gamma \in\{2$, $5, n / 10,15, n / m, n\}$. 


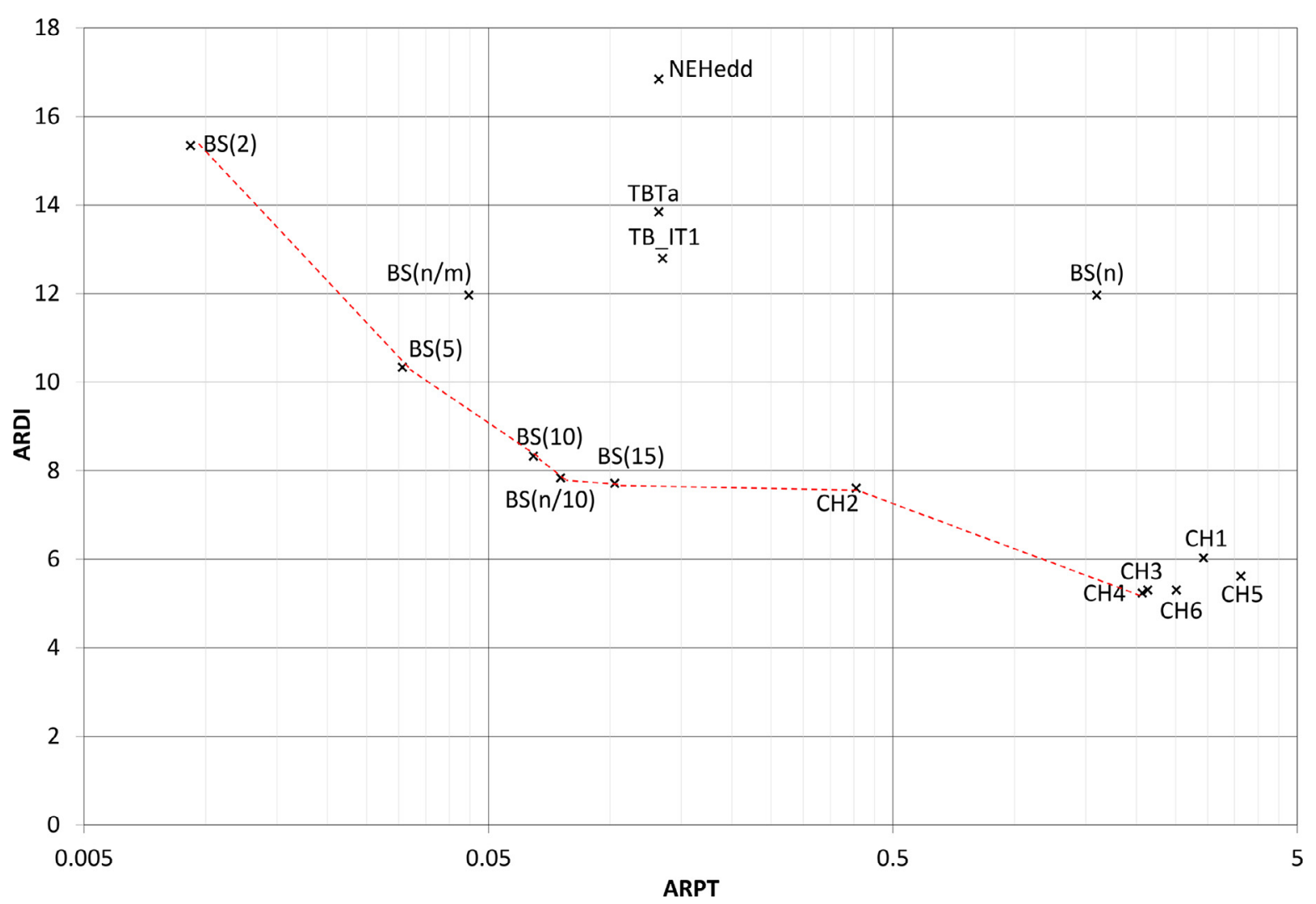

Fig. 4. ARDI1 versus ARPT for the constructive heuristics.

- Metaheuristics

- The hybrid algorithm HA proposed by Framinan and Leisten (2008).

- The genetic algorithm GAPR proposed by Vallada and Ruiz (2010).

- The evolutionary algorithm EA proposed by Cura (2015).

- The trajectory scheduling method $\mathrm{TSM}_{63}$ proposed by Li et al. (2015).

- The iterated greedy algorithm KIG proposed by Karabulut (2016).

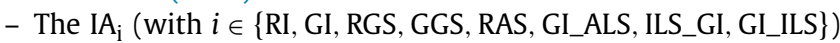
algorithms proposed in Section 3.2.

Note that the speed up procedure, proposed by Framinan and Leisten (2008), is applied in each insertion and exchange phase of all the implemented algorithms.

\subsection{Heuristics}

The computational results of the constructive heuristics are shown in Table 2, and in Fig. 4. Table 2 shows the results of the $A R D I 1_{h}$ for each heuristic $h$ grouped by $n$ and $m$. The average results in terms of $A C T_{h}, A R P T_{h}$, and $A R D I 1_{h}$ are shown in the last three rows. The dominance of each heuristic can be graphically seen in Fig. 4 (X-axis and Y-axis indicate the $A R P T_{h}$ and $A R D I 1_{h}$ of each heuristic $h$ ).

The results show that the $\mathrm{BS}(2), \mathrm{BS}(5), \mathrm{BS}(10), \mathrm{BS}(n / 10)$, and $\mathrm{BS}(15)$ algorithms are efficient for the problem (see red line in Fig. 4). To statistically support it (i.e. to discard that they are not statistically better), we perform a non-parametric Wilcoxon signedrank test for each one of the following hypotheses: $\mathrm{BS}(5)=\mathrm{BS}(\mathrm{n} / \mathrm{m})$; $\mathrm{BS}(15)=\mathrm{NEHedd} ; \mathrm{BS}(15)=\mathrm{TB}_{\mathrm{Ta}}$; and $\mathrm{BS}(15)=\mathrm{TB}_{\mathrm{IT} 1}$, where each efficient beam search algorithm has been compared against the closest heuristic. The $p$-value found for each one was .000 reject- ing each one of the previous hypotheses. In addition, several of the proposed beam search algorithms, $\mathrm{BS}(5), \mathrm{BS}(10), \mathrm{BS}(n / 10)$, and $\mathrm{BS}(15)$, clearly outperform the NEHedd and $\mathrm{TB}_{\mathrm{IT} 1}$ heuristics both in terms of ARDI1 and ARPT (or ACT). Note that, as stated in Section 1, both heuristics are the key heuristics for the problem under consideration (the NEHedd heuristic is used as initial solution for most of the algorithms developed for the problem). The excellent performance of the proposed beam search heuristic probably lies in the reduction of the complexity of the evaluation. The complexity of evaluating a full sequence in the $F m|p r m u| \Sigma T_{j}$ problem is $O(n m)$, while in the proposed algorithm it is only $O(m)$ since the jobs are inserted, one by one, at the end of a partial sequence. By reducing the complexity of this evaluation, the algorithm can evaluate much more sequences in the same CPU time. Regarding the six proposals by Li et al. (2015), which also use the NEHedd as initial solution, they perform better than each other one in terms of quality of the solution (ARDI1) but requiring much higher CPU times.

\subsection{Metaheuristics}

The re-coded algorithms (KIG, TSM $63, \mathrm{EA}, \mathrm{GAPR}$, and HA) and our proposals ( $I A_{R I}, I A_{G I}, I A_{R G S}, I A_{G G S}, I A_{R A S}, I A_{G I} A L S, I A_{I L S} G I$, and $\left.\mathrm{IA}_{\mathrm{GI} \text { ILS }}\right)$ have been run under three different stopping criteria, i.e. time equals to $60 \cdot n \cdot m, 90 \cdot n \cdot m$, and $120 \cdot n \cdot m$. The computational results for these three stopping criteria are shown, grouped by the different levels of each parameter, in Tables 3-5, respectively. These results show the good performance of GAPR against the HA metaheuristic (see hypothesis $\mathrm{H}_{1}$ in Table 6). Regarding the comparison between the last metaheuristics developed for the problem (i.e. KIG, $\mathrm{TSM}_{63}$, and $\mathrm{EA}$ ), the KIG metaheuristic clearly outperforms the other two for the three stopping criteria (hypothesis $\mathrm{H}_{2}$ ). Regarding our proposals, the following conclusions can be obtained: 
Table 3

Average $R D I 1_{h}$ of each metaheuristic for stopping criterion $60 \cdot n \cdot m$ ms grouped by the values of the parameters.

\begin{tabular}{|c|c|c|c|c|c|c|c|c|c|c|c|c|c|c|}
\hline \multicolumn{2}{|c|}{ Parameter } & \multirow{2}{*}{$\frac{\mathrm{KIG}}{0.27}$} & \multirow{2}{*}{$\begin{array}{l}\mathrm{TSM}_{63} \\
2.53\end{array}$} & \multirow{2}{*}{$\frac{E A}{2.13}$} & \multirow{2}{*}{$\begin{array}{l}\text { GAPR } \\
1.69\end{array}$} & \multirow{2}{*}{$\frac{\mathrm{HA}}{2.52}$} & \multirow{2}{*}{$\frac{\mathrm{IA}_{\mathrm{RI}}}{0.32}$} & \multirow{2}{*}{$\frac{\mathrm{IA}_{\mathrm{GI}}}{0.49}$} & \multirow{2}{*}{$\frac{\mathrm{IA}_{\mathrm{RGS}}}{0.59}$} & \multirow{2}{*}{$\frac{\mathrm{IA}_{\mathrm{GGS}}}{2.47}$} & \multirow{2}{*}{$\frac{\mathrm{IA}_{\mathrm{RAS}}}{0.27}$} & \multirow{2}{*}{$\begin{array}{l}\mathrm{IA}_{\mathrm{GI} \_A L S} \\
0.43\end{array}$} & \multirow{2}{*}{$\begin{array}{l}\text { IA }_{\text {ILS_GI }} \\
0.25\end{array}$} & \multirow{2}{*}{$\frac{\mathrm{IA}_{\mathrm{GI} \_\mathrm{ILS}}}{0.37}$} \\
\hline$T$ & 0.2 & & & & & & & & & & & & & \\
\hline$T$ & 0.4 & -0.08 & 4.39 & 4.42 & 2.87 & 5.75 & 0.02 & 0.10 & 0.56 & 3.08 & -0.43 & -0.01 & -0.23 & -0.24 \\
\hline$T$ & 0.6 & 0.13 & 6.38 & 5.07 & 3.80 & 7.98 & -0.41 & -0.62 & 0.09 & 2.02 & -1.04 & -0.70 & -0.65 & -0.87 \\
\hline$R$ & 0.2 & 0.29 & 5.94 & 4.53 & 3.47 & 7.91 & 0.02 & -0.16 & 0.47 & 2.40 & -0.60 & -0.28 & -0.37 & -0.40 \\
\hline$R$ & 0.6 & -0.05 & 4.19 & 3.80 & 2.67 & 5.17 & -0.14 & -0.13 & 0.32 & 2.63 & -0.46 & -0.17 & -0.25 & -0.37 \\
\hline$R$ & 1.0 & 0.07 & 3.17 & 3.29 & 2.22 & 3.16 & 0.05 & 0.26 & 0.45 & 2.53 & -0.13 & 0.16 & -0.02 & 0.04 \\
\hline$n$ & 50 & 0.75 & 3.97 & 3.85 & 5.47 & 3.20 & 1.12 & 1.15 & 1.77 & 7.81 & 0.81 & 1.06 & 0.83 & 0.75 \\
\hline$n$ & 150 & 0.43 & 5.36 & 5.47 & 2.81 & 5.94 & 1.19 & 1.20 & 1.70 & 3.43 & 0.53 & 1.02 & 0.90 & 0.82 \\
\hline$n$ & 250 & -0.26 & 4.56 & 3.69 & 1.43 & 6.08 & -0.68 & -0.68 & -0.29 & 0.14 & -1.01 & -0.77 & -0.83 & -0.80 \\
\hline$n$ & 350 & -0.50 & 3.84 & 2.49 & 1.43 & 6.45 & -1.72 & -1.70 & -1.53 & -1.28 & -1.93 & -1.69 & -1.75 & -1.75 \\
\hline$m$ & 10 & -0.17 & 2.08 & 0.94 & 2.02 & 3.55 & 0.26 & 0.22 & 0.49 & 1.81 & 0.07 & 0.23 & 0.23 & 0.12 \\
\hline$m$ & 30 & 0.05 & 5.02 & 4.33 & 3.07 & 5.91 & -0.26 & -0.20 & 0.21 & 2.72 & -0.63 & -0.33 & -0.50 & -0.52 \\
\hline$m$ & 50 & 0.43 & 6.19 & 6.36 & 3.27 & 6.79 & -0.07 & -0.04 & 0.53 & 3.04 & -0.64 & -0.19 & -0.37 & -0.34 \\
\hline \multicolumn{2}{|c|}{ Average } & 0.10 & 4.43 & 3.88 & 2.79 & 5.42 & -0.02 & -0.01 & 0.41 & 2.52 & -0.40 & -0.09 & -0.21 & -0.25 \\
\hline
\end{tabular}

Table 4

Average $R D I 1_{h}$ of each metaheuristic for stopping criterion $90 \cdot n \cdot m$ ms grouped by the values of the parameters.

\begin{tabular}{|c|c|c|c|c|c|c|c|c|c|c|c|c|c|c|}
\hline \multicolumn{2}{|c|}{ Parameter } & \multirow{2}{*}{$\begin{array}{l}\text { KIG } \\
0.06\end{array}$} & \multirow{2}{*}{$\begin{array}{l}\mathrm{TSM}_{63} \\
2.32\end{array}$} & \multirow{2}{*}{$\begin{array}{l}\text { EA } \\
2.00\end{array}$} & \multirow{2}{*}{$\begin{array}{l}\text { GAPR } \\
1.59\end{array}$} & \multirow{2}{*}{$\begin{array}{l}\mathrm{HA} \\
2.23\end{array}$} & \multirow{2}{*}{$\begin{array}{l}\mathrm{IA}_{\mathrm{RI}} \\
0.16\end{array}$} & \multirow{2}{*}{$\frac{\mathrm{IA}_{\mathrm{GI}}}{0.34}$} & \multirow{2}{*}{$\begin{array}{l}\mathrm{IA}_{\mathrm{RGS}} \\
0.46\end{array}$} & \multirow{2}{*}{$\frac{\mathrm{IA}_{\mathrm{GGS}}}{2.47}$} & \multirow{2}{*}{$\begin{array}{l}\mathrm{IA}_{\mathrm{RAS}} \\
0.11\end{array}$} & \multirow{2}{*}{$\begin{array}{l}\text { IA } A_{\text {GI_ALS }} \\
0.28\end{array}$} & \multirow{2}{*}{$\begin{array}{l}\mathrm{IA}_{\text {ILS_GI }} \\
0.07\end{array}$} & \multirow{2}{*}{$\begin{array}{l}\mathrm{IA}_{\mathrm{GI} \_I L S} \\
0.20\end{array}$} \\
\hline$T$ & 0.2 & & & & & & & & & & & & & \\
\hline$T$ & 0.4 & -0.58 & 4.06 & 4.20 & 2.58 & 5.24 & -0.24 & -0.15 & 0.39 & 3.07 & -0.70 & -0.30 & -0.51 & -0.50 \\
\hline$T$ & 0.6 & -0.39 & 6.02 & 4.76 & 3.40 & 7.48 & -0.64 & -0.92 & -0.06 & 2.01 & -1.31 & -0.98 & -0.95 & -1.12 \\
\hline$R$ & 0.2 & -0.28 & 5.53 & 4.25 & 3.12 & 7.31 & -0.23 & -0.43 & 0.31 & 2.40 & -0.88 & -0.56 & -0.62 & -0.71 \\
\hline$R$ & 0.6 & -0.47 & 3.90 & 3.55 & 2.40 & 4.72 & -0.34 & -0.35 & 0.19 & 2.63 & -0.69 & -0.39 & -0.54 & -0.56 \\
\hline$R$ & 1.00 & -0.16 & 2.96 & 3.16 & 2.05 & 2.93 & -0.15 & 0.05 & 0.30 & 2.52 & -0.33 & -0.04 & -0.23 & -0.15 \\
\hline$n$ & 50 & 0.52 & 3.67 & 3.69 & 5.41 & 2.85 & 0.90 & 0.91 & 1.59 & 7.81 & 0.65 & 0.80 & 0.58 & 0.62 \\
\hline$n$ & 150 & 0.06 & 4.85 & 5.32 & 2.77 & 5.50 & 0.87 & 0.83 & 1.48 & 3.43 & 0.18 & 0.70 & 0.47 & 0.44 \\
\hline$n$ & 250 & -0.73 & 4.20 & 3.39 & 0.94 & 5.44 & -0.86 & -0.89 & -0.40 & 0.13 & -1.27 & -0.97 & -1.01 & -1.03 \\
\hline$n$ & 350 & -1.06 & 3.81 & 2.22 & 0.97 & 6.16 & -1.87 & -1.82 & -1.61 & -1.30 & -2.09 & -1.85 & -1.90 & -1.94 \\
\hline$m$ & 10 & -0.33 & 1.95 & 0.84 & 1.83 & 3.29 & 0.14 & 0.09 & 0.42 & 1.79 & -0.07 & 0.11 & 0.14 & -0.03 \\
\hline$m$ & 30 & -0.41 & 4.68 & 4.05 & 2.80 & 5.44 & -0.48 & -0.44 & 0.03 & 2.72 & -0.88 & -0.57 & -0.82 & -0.78 \\
\hline$m$ & 50 & -0.17 & 5.76 & 6.07 & 2.94 & 6.22 & -0.38 & -0.38 & 0.33 & 3.04 & -0.95 & -0.53 & -0.71 & -0.62 \\
\hline \multicolumn{2}{|c|}{ Average } & -0.30 & 4.13 & 3.65 & 2.52 & 4.99 & -0.24 & -0.24 & 0.26 & 2.52 & -0.63 & -0.33 & -0.47 & -0.47 \\
\hline
\end{tabular}

Table 5

Average $R D I 1_{h}$ of each metaheuristic $h$ for stopping criterion $120 \cdot n \cdot m$ ms grouped by the values of the parameters.

\begin{tabular}{|c|c|c|c|c|c|c|c|c|c|c|c|c|c|c|}
\hline \multicolumn{2}{|c|}{ Parameter } & \multirow{2}{*}{$\frac{\mathrm{KIG}}{-0.08}$} & \multirow{2}{*}{$\frac{\mathrm{TSM}_{63}}{2.14}$} & \multirow{2}{*}{$\frac{E A}{1.92}$} & \multirow{2}{*}{$\begin{array}{l}\text { GAPR } \\
1.52\end{array}$} & \multirow{2}{*}{$\frac{\mathrm{HA}}{2.02}$} & \multirow{2}{*}{$\frac{\mathrm{IA}_{\mathrm{RI}}}{0.05}$} & \multirow{2}{*}{$\frac{\mathrm{IA}_{\mathrm{Gl}}}{0.25}$} & \multirow{2}{*}{$\frac{I A_{\text {RGS }}}{0.38}$} & \multirow{2}{*}{$\frac{\mathrm{IA}_{\mathrm{GGS}}}{2.47}$} & \multirow{2}{*}{$\begin{array}{l}\mathrm{IA}_{\mathrm{RAS}} \\
0.00\end{array}$} & \multirow{2}{*}{$\begin{array}{l}\text { IA } A_{\text {GI_ALS }} \\
0.19\end{array}$} & \multirow{2}{*}{$\frac{I_{\text {ILS_GI }}}{-0.01}$} & \multirow{2}{*}{$\frac{\mathrm{IA}_{\mathrm{GI} \_\mathrm{ILS}}}{0.10}$} \\
\hline$T$ & 0.2 & & & & & & & & & & & & & \\
\hline$T$ & 0.4 & -0.89 & 3.88 & 4.11 & 2.42 & 4.99 & -0.39 & -0.34 & 0.24 & 3.06 & -0.84 & -0.46 & -0.65 & -0.67 \\
\hline$T$ & 0.6 & -0.72 & 5.72 & 4.54 & 3.20 & 7.20 & -0.80 & -1.09 & -0.21 & 2.01 & -1.52 & -1.19 & -1.11 & -1.30 \\
\hline$R$ & 0.2 & -0.62 & 5.25 & 4.07 & 2.89 & 6.97 & -0.39 & -0.62 & 0.20 & 2.39 & -1.07 & -0.75 & -0.75 & -0.88 \\
\hline$R$ & 0.6 & -0.68 & 3.71 & 3.40 & 2.27 & 4.50 & -0.47 & -0.47 & 0.02 & 2.62 & -0.86 & -0.55 & -0.67 & -0.71 \\
\hline$R$ & 1.00 & -0.38 & 2.79 & 3.10 & 1.98 & 2.74 & -0.28 & -0.09 & 0.19 & 2.52 & -0.44 & -0.17 & -0.35 & -0.27 \\
\hline$n$ & 50 & 0.40 & 3.40 & 3.59 & 5.41 & 2.69 & 0.79 & 0.76 & 1.42 & 7.81 & 0.54 & 0.66 & 0.47 & 0.54 \\
\hline$n$ & 150 & -0.20 & 4.48 & 5.25 & 2.77 & 5.28 & 0.60 & 0.60 & 1.32 & 3.43 & -0.05 & 0.48 & 0.26 & 0.20 \\
\hline$n$ & 250 & -1.04 & 3.99 & 3.26 & 0.69 & 5.16 & -0.97 & -1.04 & -0.52 & 0.13 & -1.44 & -1.13 & -1.14 & -1.18 \\
\hline$n$ & 350 & -1.41 & 3.79 & 2.00 & 0.66 & 5.82 & -1.94 & -1.89 & -1.67 & -1.31 & -2.21 & -1.97 & -1.96 & -2.04 \\
\hline$m$ & 10 & -0.41 & 1.85 & 0.77 & 1.76 & 3.13 & 0.09 & 0.00 & 0.33 & 1.78 & -0.15 & 0.02 & 0.07 & -0.11 \\
\hline$m$ & 30 & -0.72 & 4.42 & 3.88 & 2.67 & 5.17 & -0.67 & -0.61 & -0.09 & 2.72 & -1.06 & -0.78 & -0.98 & -0.93 \\
\hline$m$ & 50 & -0.55 & 5.47 & 5.92 & 2.71 & 5.91 & -0.56 & -0.57 & 0.17 & 3.03 & -1.16 & -0.70 & -0.87 & -0.82 \\
\hline \multicolumn{2}{|c|}{ Average } & -0.56 & 3.91 & 3.52 & 2.38 & 4.74 & -0.38 & -0.39 & 0.14 & 2.51 & -0.79 & -0.49 & -0.59 & -0.62 \\
\hline
\end{tabular}

1. The random adjacent swap is the best perturbation among our eight proposals, for all time limits tested (hypothesis $\mathrm{H}_{3}$ ).

2. In addition, the $I A_{R A S}$ is efficient and outperforms each other metaheuristic for the problem (hypothesis $\mathrm{H}_{4}$ ).

3. The iterated algorithm based on greedy general swap (IA $\mathrm{GGS}_{\text {) }}$ performs worst than each other perturbation method (hypothesis $\mathrm{H}_{5}$ ).

4. Similarly as in the $F m|p r m u| C_{\max }$ (Dubois-Lacoste et al., 2017), the greedy insertion plus local search insertion(IA ILS_GI $_{\text {I }}$ ) outperforms the greedy insertion (hypothesis $\mathrm{H}_{6}$ ).

5. The random and greedy insertions $\left(\mathrm{IA}_{\mathrm{RI}}\right.$ and $\mathrm{IA}_{\mathrm{GI}}$, respectively) perform very similar (hypothesis $\mathrm{H}_{7}$ ).

To justify each previous conclusion, the following hypotheses are checked for statistical evidence: $G A P R=H A\left(H_{1}\right) ; K I G=E A$

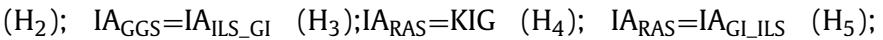

$I_{A_{G I} I L S}=I A_{G I}\left(H_{6}\right)$; and $I_{R I}=I_{G I}\left(H_{7}\right)$. Results are shown in Table 6 for stopping criterion $60 \cdot n \cdot m$ (the same statistical evidences have been found for the other parameters). The last two columns show the results obtained using Holm's procedure (see e.g. Pan, Tasgetiren, and Liang, 2008 and Fernandez-Viagas and Framinan, 2015b for related studies). No statistical evidence has been found only for the hypothesis that the random insertion outperforms the greedy insertion. In addition, it is worth highlighting that the excellent performance of $I_{\mathrm{RAS}}$ probably lies in performing several small variations in the sequence to decrease the number of "bad" solutions evaluated. The perturbation phase of this iterated algorithm is performed over a sequence which is a local optimum, therefore this sequence is presumably "good" and introducing a high number of changes in this sequence seems to produce, in many cases, sequences that are worse than the ini- 
Table 6

Holm's procedure.

\begin{tabular}{llllll}
\hline $\mathrm{H}_{i}$ & Hypothesis & $p$-value & Wilcoxon & $\alpha /(7-i+1)$ & Holm's procedure \\
\hline $\mathrm{H}_{1}$ & $\mathrm{GAPR}=\mathrm{HA}$ & .000 & $\mathrm{R}$ & 0.0071 & $\mathrm{R}$ \\
$\mathrm{H}_{2}$ & $\mathrm{KIG}=\mathrm{EA}$ & .000 & $\mathrm{R}$ & 0.0083 & $\mathrm{R}$ \\
$\mathrm{H}_{3}$ & $\mathrm{IA}_{\mathrm{RAS}}=\mathrm{I} \mathrm{I}_{\mathrm{GL} \_\mathrm{ILS}}$ & .000 & $\mathrm{R}$ & 0.0100 & $\mathrm{R}$ \\
$\mathrm{H}_{4}$ & $\mathrm{IA}_{\mathrm{RAS}}=\mathrm{KIG}$ & .000 & $\mathrm{R}$ & 0.0125 & $\mathrm{R}$ \\
$\mathrm{H}_{5}$ & $\mathrm{IA}_{\mathrm{GGS}}=\mathrm{I} \mathrm{I}_{\mathrm{RGS}}$ & .000 & & 0.0167 & \\
$\mathrm{H}_{6}$ & $\mathrm{IA}_{\mathrm{ILS} \text { GII }}=\mathrm{IA} \mathrm{A}_{\mathrm{GI}}$ & .000 & & 0.0250 & \\
$\mathrm{H}_{7}$ & $\mathrm{IA}_{\mathrm{RI}}=\mathrm{IA} \mathrm{A}_{\mathrm{GI}}$ & .939 & & 0.0500 & \\
\hline
\end{tabular}

tial, but that have to computed, thus wasting CPU effort. Similar results have been found for example both in Rad, Ruiz, and Boroojerdian (2009) and Fernandez-Viagas and Framinan (2015d), which could lead to similar conclusions.

\section{Conclusions}

In this paper we have proposed two different sets of algorithms to solve the permutation flow shop scheduling problem to minimise the total tardiness. Firstly, we have proposed a set of beamsearch-based heuristics varying the size of their population. These are fast heuristics that construct solutions by adding jobs at the end of several partial sequences constructed in parallel. In addition, this set uses properties of the problem both to estimate the performance of each partial sequence and to be able to compare sequences with different jobs. Secondly, we have proposed several simple iterated-greedy-based algorithms with several types of destruction-construction phases. The methods developed to perturb the solutions are based on insertion, general swap, adjacent swap, and partial local searches.

Our proposals have been compared with the state-of-the-art algorithms of the problem under study in a well-known benchmark testbed. More specifically, a total of 14 algorithms have been reimplemented and compared with our proposals (a set of beam search algorithms varying the size of the population, and eight different iterated algorithms). Regarding constructive heuristics, the results show that BS(15) clearly outperforms the NEHedd in terms of quality of solutions and computational effort. In addition, the proposed heuristics $\mathrm{BS}(2), \mathrm{BS}(5), \mathrm{BS}(10), \mathrm{BS}(n / 10)$, and $\mathrm{BS}(15)$ are efficient heuristics for the problem. Regarding the computational evaluation of metaheuristics, the iterated algorithm with a simple random adjacent swap ( $I A_{\text {RAS }}$ ) clearly outperforms the other seven simple and complex perturbation methods of the iterated algorithm, and statistically outperforms each other existing metaheuristic for the problem under study.

Due to the excellent performance of the original iterated greedy in different scheduling problems, it is noteworthy to mention that the conclusions obtained by applying the simple random adjacent swap, such as the destruction-construction phase of the proposed iterated algorithm, could probably be extended for future iteratedgreedy-based algorithms developed for either the problem under consideration, or for related scheduling problems.

\section{Acknowledgements}

This research has been funded by the Spanish Ministry of Science and Innovation, under projects "ADDRESS" with reference DPI2013-44461-P and "PROMISE" with reference DPI2016-80750-P.

\section{Supplementary material}

Supplementary material associated with this article can be found, in the online version, at 10.1016/j.eswa.2017.10.050

\section{References}

Cura, T. (2015). An evolutionary algorithm for the permutation flowshop scheduling problem with total tardiness criterion. International Journal of Operational Research, 22(3), 366-384. doi:10.1504/IJOR.2015.068287.

Dong, X., Huang, H., \& Chen, P. (2009). An iterated local search algorithm for the permutation flowshop problem with total flowtime criterion. Computers \& Operations Research, 36(5), 1664-1669.

Du, J., \& Leung, J. (1990). Minimizing total tardiness on one machine is np-hard. Mathematics of Operations Research, 15(3), 483-495.

Dubois-Lacoste, J., Pagnozzi, F., \& Stützle, T. (2017). An iterated greedy algorithm with optimization of partial solutions for the makespan permutation flowshop problem. Computers and Operations Research, 81, 160-166. doi:10.1016/j.cor.2016. 12.021.

Fernandez-Viagas, V., Dios, M., \& Framinan, J. (2016a). Efficient constructive and composite heuristics for the permutation flowshop to minimise total earliness and tardiness. Computers and Operations Research, 75, 38-48. doi:10.1016/j.cor. 2016.05.006.

Fernandez-Viagas, V., \& Framinan, J. (2015a). A bounded-search iterated greedy algorithm for the distributed permutation flowshop scheduling problem. International Journal of Production Research, 53(4), 1111-1123.

Fernandez-Viagas, V., \& Framinan, J. (2015b). Efficient non-population-based algorithms for the permutation flowshop scheduling problem with makespan minimisation subject to a maximum tardiness. Computers $\mathcal{E}$ Operations Research, 64(0), 86-96. https://doi.org/10.1016/j.cor.2015.05.006.

Fernandez-Viagas, V., \& Framinan, J. (2015c). A new set of high-performing heuristics to minimise flowtime in permutation flowshops. Computers \& Operations Research, 53, 68-80.

Fernandez-Viagas, V., \& Framinan, J. (2015d). NEH-based heuristics for the permutation flowshop scheduling problem to minimise total tardiness. Computers $\mathcal{E}$ Operations Research, 60, 27-36. doi:10.1016/j.cor.2015.02.002.

Fernandez-Viagas, V., \& Framinan, J. (2017). A beam-search-based constructive heuristic for the pfsp to minimise total flowtime. Computers and Operations Research, 81, 167-177. doi:10.1016/j.cor.2016.12.020.

Fernandez-Viagas, V., \& Framinan, J. M. (2014). On insertion tie-breaking rules in heuristics for the permutation flowshop scheduling problem. Computers $\&$ Operations Research, 45(0), 60-67. https://doi.org/10.1016/j.cor.2013.12.012.

Fernandez-Viagas, V., Leisten, R., \& Framinan, J. (2016b). A computational evaluation of constructive and improvement heuristics for the blocking flow shop to minimise total flowtime. Expert Systems with Applications, 61, 290-301. doi:10.1016 j.eswa.2016.05.040.

Fernandez-Viagas, V., Ruiz, R., \& Framinan, J. (2017). A new vision of approximate methods for the permutation flowshop to minimise makespan: State-of-the-art and computational evaluation. European Journal of Operational Research, 257(3), 707-721. doi:10.1016/j.ejor.2016.09.055.

Framinan, J., \& Leisten, R. (2008). Total tardiness minimization in permutation flow shops: A simple approach based on a variable greedy algorithm. International Journal of Production Research, 46(22), 6479-6498.

Hasija, S., \& Rajendran, C. (2004). Scheduling in flowshops to minimize total tardiness of jobs. International Journal of Production Research, 42(11), 2289-2301.

Karabulut, K. (2016). A hybrid iterated greedy algorithm for total tardiness minimization in permutation flowshops. Computers and Industrial Engineering, 98, 300-307. doi:10.1016/j.cie.2016.06.012.

Kim, Y.-D. (1993). Heuristics for flowshop scheduling problems minimizing mean tardiness. Journal of the Operational Research Society, 44(1), 19-28.

Kim, Y.-D., Lim, H.-G., \& Park, M.-W. (1996). Search heuristics for a flowshop scheduling problem in a printed circuit board assembly process. European Journal of Operational Research, 91(1), 124-143.

Li, X., Chen, L., Xu, H., \& Gupta, J. (2015). Trajectory scheduling methods for minimizing total tardiness in a flowshop. Operations Research Perspectives, 2, 13-23. doi:10.1016/j.orp.2014.12.001.

Li, X., Wang, Q., \& Wu, C. (2009). Efficient composite heuristics for total flowtime minimization in permutation flow shops. OMEGA, The International Journal of Management Science, 37(1), 155-164.

Liu, J., \& Reeves, C. (2001). Constructive and composite heuristic solutions to the $P \| \Sigma c_{i}$ scheduling problem. European Journal of Operational Research, 132, 439-452.

Naderi, B., \& Ruiz, R. (2010). The distributed permutation flowshop scheduling problem. Computers \& Operations Research, 37(4), 754-768.

Nawaz, M., Enscore, E., Jr., \& Ham, I. (1983). A heuristic algorithm for the m-ma- 
chine, n-job flow-shop sequencing problem. OMEGA, The International Journal of Management Science, 11(1), 91-95.

Pan, Q-K. Gao, L. Li, X.-Y., \& Gao, K.-Z. (2017). Effective metaheuristics for scheduling a hybrid flowshop with sequence-dependent setup times. Applied Mathematics and Computation, 303, 89-112. doi:10.1016/j.amc.2017.01.004.

Pan, Q.-K. \& Ruiz, R. (2013). A comprehensive review and evaluation of permutation flowshop heuristics to minimize flowtime. Computers \&' Operations Research, 40(1), 117-128.

Pan, Q.-K., \& Ruiz, R. (2014). An effective iterated greedy algorithm for the mixed no-idle permutation flowshop scheduling problem. Omega (United Kingdom), 44, 41-50. doi:10.1016/j.omega.2013.10.002.

Pan, Q.-K., Tasgetiren, M., \& Liang, Y.-C. (2008). A discrete differential evolution algorithm for the permutation flowshop scheduling problem. Computers and Industrial Engineering, 55(4), 795-816.

Panwalkar, S., Smith, M., \& Seidmann, A. (1982). Common due date assignment to minimize total penalty for the one machine scheduling problem.. Operations Research, 30(2), 391-399.

Parthasarathy, S., \& Rajendran, C. (1997). A simulated annealing heuristic for scheduling to minimize mean weighted tardiness in a flowshop with sequencedependent setup times of jobs-a case study. Production Planning and Control, 8(5), 475-483. doi:10.1080/095372897235055.

Parthasarathy, S., \& Rajendran, C. (1998). Scheduling to minimize mean tardiness and weighted mean tardiness in flowshop and flowline-based manufacturing cell. Computers and Industrial Engineering, 34(2-4), 531-546.

Pinedo, M. (1995). Scheduling: Theory, algorithms and systems. Prentice Hall.

Potts, C., \& Van Wassenhove, L. (1982). A decomposition algorithm for the single machine total tardiness problem. Operations Research Letters, 1(5), 177-181.

Rad, S. F., Ruiz, R., \& Boroojerdian, N. (2009). New high performing heuristics for minimizing makespan in permutation flowshops. OMEGA, The International Journal of Management Science, 37(2), 331-345.

Raman, N. (1995). Minimum tardiness scheduling in flow shops: Construction and evaluation of alternative solution approaches. Journal of Operations Management, 12(2), 131-151.
Ribas, I., Companys, R., \& Tort-Martorell, X. (2017). Efficient heuristics for the parallel blocking flow shop scheduling problem. Expert Systems with Applications, 74 41-54. doi:10.1016/j.eswa.2017.01.006.

Rios-Mercado, R., \& Bard, J. (1998). Heuristics for the flow line problem with setup costs. European Journal of Operational Research, 110(1), 76-98.

Ruiz, R., \& Maroto, C. (2005). A comprehensive review and evaluation of permutation flowshop heuristics. European Journal of Operational Research, 165(2), 479-494.

Ruiz, R., \& Stützle, T. (2007). A simple and effective iterated greedy algorithm for the permutation flowshop scheduling problem. European Journal of Operational Research, 177(3), 2033-2049.

Sen, T., \& Gupta, S. (1984). A state-of-art survey of static scheduling research involving due dates. OMEGA, The International Journal of Management Science, 12(1), 63-76.

Taillard, E. (1990). Some efficient heuristic methods for the flow shop sequencing problem. European Journal of Operational Research, 47(1), 65-74.

Taillard, E. (1993). Benchmarks for basic scheduling problems. European Journal of Operational Research, 64(2), 278-285.

Valente, J. (2010). Beam search heuristics for quadratic earliness and tardiness scheduling. Journal of the Operational Research Society, 61(4), 620-631. doi:10. 1057 /jors.2008.191.

Valente, J., \& Alves, R. (2005). Filtered and recovering beam search algorithms for the early/tardy scheduling problem with no idle time. Computers and Industrial Engineering, 48(2), 363-375. doi:10.1016/j.cie.2005.01.020.

Valente, J., \& Alves, R. (2008). Beam search algorithms for the single machine total weighted tardiness scheduling problem with sequence-dependent setups. Computers and Operations Research, 35(7), 2388-2405.

Vallada, E., \& Ruiz, R. (2010). Genetic algorithms with path relinking for the minimum tardiness permutation flowshop problem. OMEGA, The International Journal of Management Science, 38(1-2), 57-67.

Vallada, E., Ruiz, R., \& Minella, G. (2008). Minimising total tardiness in the m-machine flowshop problem: A review and evaluation of heuristics and metaheuristics. Computers \& Operations Research, 35(4), 1350-1373.

Zanakis, S., Evans, J., \& Vazacopoulos, A. (1989). Heuristic methods and applications: A categorized survey. European Journal of Operational Research, 43(1), 88-110.

Zäpfel, G., Braune, R., \& Bögl, M. (2010). Metaheuristic search concepts. Springer. 